\title{
Melt flow characteristics in gas-assisted laser cutting
}

\author{
B TIRUMALA RAO and A K NATH
}

Centre for Advanced Technology, Indore 452 013, India

email: trao@cat.ernet.in

MS received 24 May 2001; revised 28 December 2001

\begin{abstract}
We present a study on laser cutting of mild steel with oxygen as an assist gas. We correlate the cut surface quality with the melt film thickness. We estimate the optimum pressure required for melt ejection under laminar flow regime. The thickness of melt film inside the kerf is estimated using mass balance and the shear force acting on the cutting front assuming melt flow profile as linear. The dependence of melt film thickness on gas pressure, cutting velocity and work piece thickness is estimated and compared with experimental results.
\end{abstract}

Keywords. Laser cutting; gas flow; shear stress; melt film thickness.

\section{Introduction}

Carbon dioxide $\left(\mathrm{CO}_{2}\right)$ laser cutting of thick mild steel with oxygen is one of the evolving industrial applications. In this process the cut surface quality is very important. The basic parameters in laser cutting are cutting speed, kerf width, laser power, chemistry of gas, nozzle exit pressure, nozzle design, workpiece thickness and surface quality. In oxygen assist laser cutting, the gas jet plays two roles, one is to generate additional thermal energy during formation of $\mathrm{FeO}$ and other oxides. This additional energy enhances the process parameters like cutting speed and thickness of the workpiece. The second role is to supply the shear force to the gas/liquid boundary to eject the molten metal formed during the cutting process. In laser cutting the major process is the instantaneous melt ejection, which is achieved by selecting appropriate operating window of the cutting speed, gas pressure, laser power, and stand-off distance etc.,

The cut surface quality is influenced by the melt film thickness developed inside the kerf during the process, it will be poor for larger melt thickness. Therefore it is important to understand how various experimental parameters influence the melt film thickness. Although much work has been done in this direction the phenomenon of melt ejection is poorly defined due to its complex nature. The melt film thickness is dependent on the $\mathrm{FeO}$ formation, since $\mathrm{FeO}$ melt has lower viscosity compared to the Fe melt. The fraction of $\mathrm{FeO}$ formation reduces with increasing cutting speed up to a certain limit and then reaches a steady value as reported by Kaplan (1996). Yilbas \& Kar (1998) reported a similar trend in the variation of melt film thickness with assist-gas velocity.

A list of symbols is given at the end of the paper 
The present study is confined to gas-assisted cutting with the convergent nozzle and very small stand-off distance. The work does not deal with the thermodynamics of melt formation or heat flow into the work piece. The objective of the present work is to estimate the optimum pressure required for melt ejection under laminar flow regime, using a uniform flow over a flat plate (Landau \& Lifshitz 1987; Kumar 1988). Also the melt film thickness inside the kerf during the process is estimated by the analytical model taking into account the mass balance of molten metal coupled with shear force required to drag the molten film. The dependence of the gas pressure, cutting speed and workpiece thickness on melt film thickness is modelled and compared with the experimental results.

\section{Model description}

The laser-cutting setup is shown in the figure 1. A fraction of the gas coming out of the converging nozzle exit enters into the kerf with a constant velocity (Shapiro 1953; Liepmann \& Roshko 1957) independent of the stagnation pressure provided that the nozzle exit is maintained at the critical state. The nozzle exit is said to be in critical state, when the ratio of ambient to stagnation pressure is less than 0.5283 (Shapiro 1953; Liepmann \& Roshko 1957). In the critical state the exit gas velocity would be $301 \mathrm{~m} / \mathrm{s}$ for $\mathrm{O}_{2}$ at stagnation temperature of $300 \mathrm{~K}$ (Shapiro 1953; Liepmann \& Roshko 1957). We assume that nozzle stand-off distance is very small, the melt surface is flat, the kerf width is very large $(>500 \mu \mathrm{m})$ compared to the gaseous boundary layer $(\sim 50 \mu \mathrm{m})$ (Kumar 1988; Heidenreich et al 1996) and the variation of the gas density is very small. Under these assumptions the boundary layer theory flow over

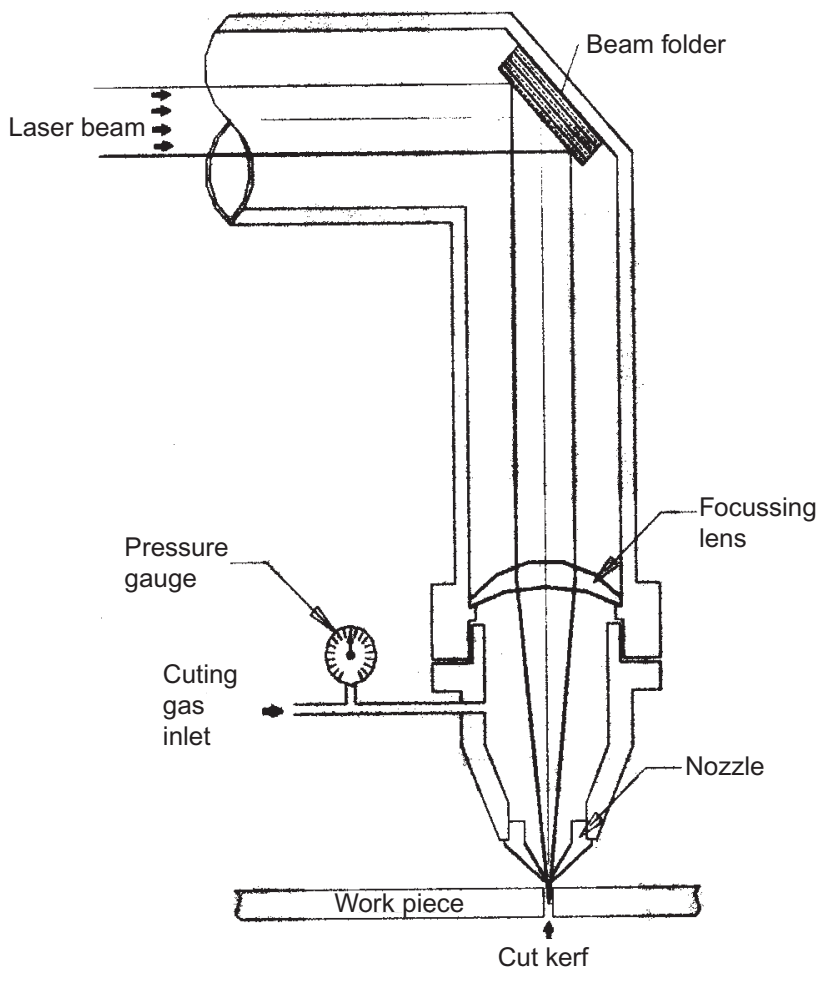

Figure 1. Laser cutting setup. 
a flat plate can be applied to estimate the distance after which the flow separation takes place by calculating the critical Reynolds number (Landau \& Lifshitz 1987; Kumar 1988),

$$
\operatorname{Re}_{z}=U Z \rho / \mu \text {. }
$$

This is because a laminar layer tends to separate off the surface more readily than a turbulent layer (Kumar 1988). When the value of $\mathrm{Re}_{z}$ exceeds $3.2 \times 10^{5}$ the gas flow suffers a change from laminar to turbulent; this is called the separation point (Landau \& Lifshitz 1987; Kumar 1988; Heidenreich et al 1996). At this separation point the shear stress exerting on the melt is significantly reduced due to wake formation, which results in inefficient melt ejection and thicker molten layer. Also in this region the diffusion of oxygen in Fe changes due to turbulence, consequently this develops poor cut quality. To achieve more or less uniform cut quality the gas flow in the kerf should be laminar in nature. Using the critical Reynolds number at the separation and relations amongst the critical gas pressure, density and temperature (Shapiro 1953; Liepmann \& Roshko 1957) the following relation gives the depth of the cut after which the gas flow tends to separate with respect to the stagnation gas pressure.

$$
Z=22.5 / P_{0}
$$

From this relation we can find the nozzle pressure for a given thickness $d$ of the sheet, so the flow should be laminar throughout the depth, for which $Z=d$. The above relationship is valid closely for the larger kerf width, since the length of the laminar gas flow inside the kerf is dependent on the kerf width (Heidenreich et al 1996). If the kerf width is small then the value of $Z$ reduces significantly.

The shear force acting on the melt film at the gas / liquid interface is (Vicanek \& Simon 1987; Kumar 1988; Yilbas \& Kar 1998),

$$
\tau=0.332\left(\rho U^{2}\right) / \overline{\mathrm{R}} \mathrm{e}_{z}^{1 / 2}
$$

The present model balances this shear force with the melt viscous forces. Since the thickness of the melt film is very small (Yilbas \& Kar 1998), we assume that melt velocity profile is linear (figure 2). The maximum melt velocity $u_{\max }$ is at gas/melt interface and zero melt

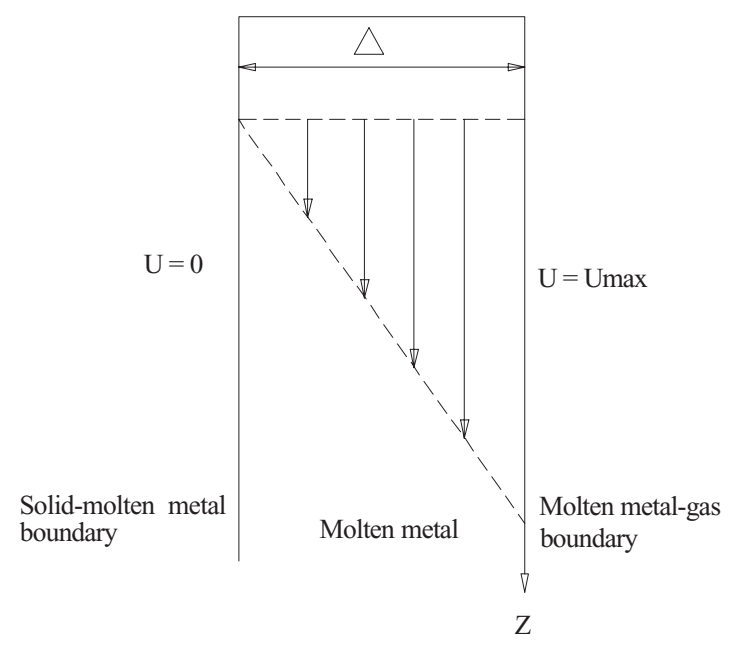

Figure 2. Melt velocity profile. 
velocity is at melt/solid interface. If $u_{\mathrm{avg}}$ is the melt average velocity, which is $u_{\max } / 2$, and melt is ejected due to the shear force given by the gas jet, then

$$
\tau=\mu_{m}\left(u_{\max } / \Delta\right) .
$$

From this we get $u_{\mathrm{avg}}=\tau \Delta / 2 \mu_{m}$.

Mass balance criteria:the amount of molten mass in per unit time $=\rho_{m} v_{c} \mathrm{~d} w$,

the amount of molten mass out per unit time $=\rho_{m} u_{\mathrm{avg}} \Delta w$.

In the steady state: molten mass in per unit time $=$ molten mass out per unit time.

Also by substituting melt average velocity (4) in the equation we get the melt film thickness

$$
\Delta=\left(2 \mu_{m} v_{c} Z / \tau\right)^{1 / 2} .
$$

This relation gives the dependence of melt film thickness on gas pressure, cutting speed and workpiece thickness.

\section{Experimental results and discussions}

Reynolds number increases at any given depth with gas pressure, (1). The extent of laminar flow in the kerf is dependent on stagnation gas pressure in the nozzle. Maintaining the gas flow in the laminar regime, the operating range of pressure can be large in thin sheet cutting. Figure 3 shows the stagnation gas pressure that can sustain laminar gas flow in the entire thickness of the workpiece under the assumption that the nozzle stand-off distance is $0.5 \mathrm{~mm}$ and the kerf width is about $\sim 1 \mathrm{~mm}$. Since the online measurement of melt film thickness is beyond the scope of the present experimental study, we try to estimate it using the striation wavelength. Since striation wavelength is decided by surface tension effects of the melt, oxidation front propagation speed and laser beam diameter at the metal surface, besides the cut speed and gas flow velocity, the estimated melt film thickness from the striation wavelength is expected to be on the higher side. Figure 4 shows the variation of melt film thickness with the thickness of the workpiece at constant cutting velocity and different stagnation gas pressures. Operating at higher gas pressure results in minimum melt film thickness sustained on the cut surface. Figure 5 shows the variation of melt film thickness with workpiece thickness for different cutting velocities at constant gas pressure. When operated at higher cutting velocity thick melt film sustains as very coarse striations on the cut surface. Experimental values are also plotted on the same figures. While the experimental and theoretical trends of variations match well, the discrepancy in actual values could be due to the overestimation of experimental values of thickness as mentioned above.

Also the impact of cutting speed on the melt film thickness (at same laser power $600 \mathrm{~W}$ and gas pressure $1 \mathrm{bar}=10^{5} \mathrm{~N} / \mathrm{m}^{2}$ ) can be seen in figure 6 . Figures $6 \mathrm{a}$ and $\mathrm{b}$ depict the laser cut surfaces of $5 \mathrm{~mm}$ thick mild steel plate when cutting is done at $500 \mathrm{~mm} / \mathrm{min}$ and $1000 \mathrm{~mm} / \mathrm{min}$ speeds respectively. The one with higher cutting speed has coarse striations indicating the existence of thicker melt film during the cutting. Figure 7 shows the laser cut surface of $5 \mathrm{~mm}$ thick mild steel plate with gas pressure of 3 bar and cutting speed of $500 \mathrm{~mm} / \mathrm{min}$. 

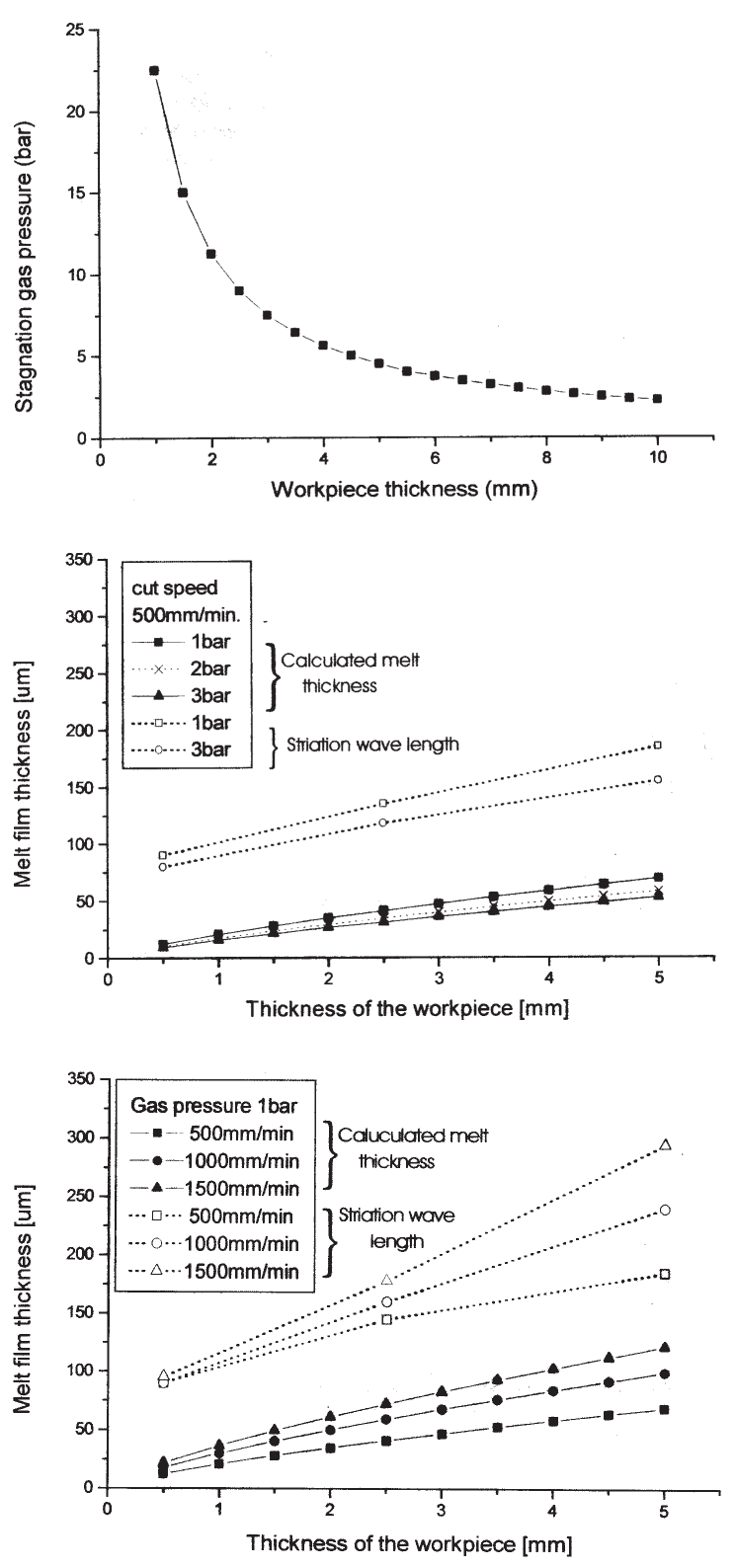

Figure 3. Stagnation gas pressure vs workpiece thickness.

Figure 4. Melt film thickness variation with gas pressure.

Figure 5. Melt film thickness variation with cutting velocity

(a)

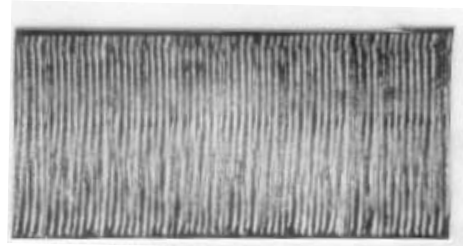

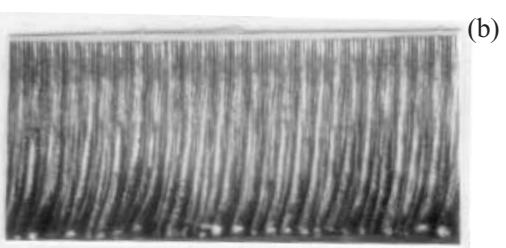

Figure 6. Laser cut surface of MS $(5 \mathrm{~mm})$ : (a) speed $=500 \mathrm{~mm} / \mathrm{min}$, pressure $=1$ bar; (b) speed $=1000 \mathrm{~mm} / \mathrm{min}$, pressure $=1$ bar. 


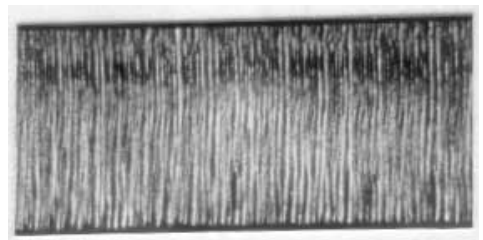

Figure 7. Laser cut surface of MS (5 mm) (a) speed = $500 \mathrm{~mm} / \mathrm{min}$, pressure $=1 \mathrm{bar}$.

The impact of change in gas pressure on the melt film thickness is not as much as that of cutting speed. The same is evident form figure 4 (change in gas pressure) and figure 5 (change in cutting velocity), and also from the cut surfaces shown in figures 6a and 7 (change in gas pressure), and figures $6 \mathrm{a}$ and $\mathrm{b}$ (change in cutting velocity.). For $5 \mathrm{~mm}$ thick mild steel plates at $600 \mathrm{~W}$ laser power and one bar gas pressure the maximum cutting speed is $1300 \mathrm{~mm} / \mathrm{min}$, and when operated at 3 bar gas pressure the optimum cutting speed is $1500 \mathrm{~mm} / \mathrm{min}$ with good cut edge quality. Beyond these speeds the bottom part of the cut is attached with the dross.

Figure 8 a shows the laser cut surface of $10 \mathrm{~mm}$ thick mild steel plate when cutting is done at 1.5 bar. It is found that during cutting at higher gas pressure $(3 \mathrm{bar})$ the lower part of the cut surface is badly damaged as shown in figure $8 \mathrm{~b}$. This could be due to turbulent gas flow as explained earlier.

\section{Conclusions}

To achieve minimum melt film thickness on the cut surface laser cutting should be performed at optimum cutting velocity and maximum gas pressure such that laminar gas flow is sustained in the entire kerf depth.

For a sufficiently large kerf width in thin sheet cutting the operating range of gas pressure is more than that in thick sheet cutting, as laminar flow can be maintained throughout the depth of the cut in thin sheets. In other words, thin sheets can be cut with an optimum gas pressure and a smaller kerf width with laminar gas flow.

To cut thick sheets, the focal point of the beam should be inside the workpiece so that it provides larger kerf width, which allows the flow to be laminar up to larger depths. To achieve

(a)

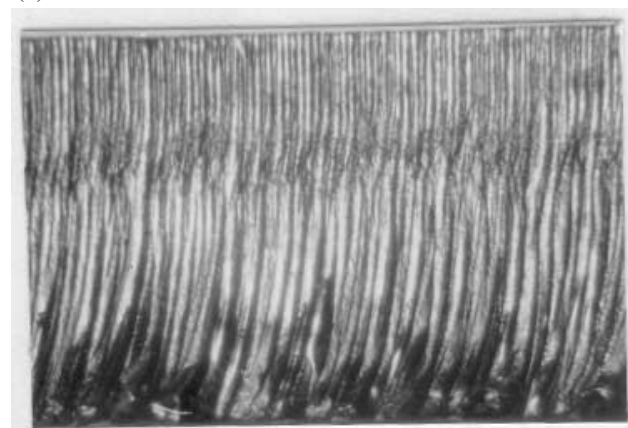

(b)

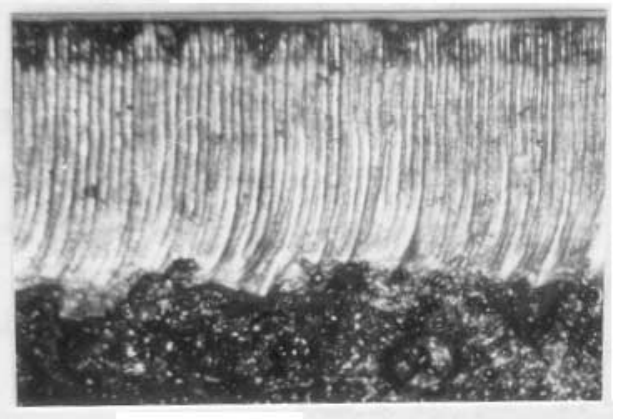

Figure 8. Laser cut surface of MS $(10 \mathrm{~mm})$ : (a) speed $=300 \mathrm{~mm} / \mathrm{min}$, pressure $=1$ bar; (b) speed $=300 \mathrm{~mm} / \mathrm{min}$, pressure $=3 \mathrm{bar}$. 
better cut quality in thick plates $(>10 \mathrm{~mm})$, the operating pressure should be less than 2 bar so that laminar gas flow is sustained in the entire depth.

The melt film thickness, which decides the roughness of the cut surface, is influenced to a greater extent by the change in cutting velocity than the variation in the cutting nozzle gas pressure.

The authors would like to thank R Sridhar and C H Prem Singh for their kind assistance in the experiments.

\section{List of symbols}

$d \quad$ thickness of the work piece (m);

$P_{0} \quad$ stagnation gas pressure (bar, 1 bar $=10^{5} \mathrm{~N} / \mathrm{m}^{2}$ );

$s \quad$ nozzle stand-off distance $(\mathrm{m})$;

$U \quad$ gas jet velocity $(\mathrm{m} / \mathrm{s})$;

$u_{\text {avg }}$ average melt velocity $(\mathrm{m} / \mathrm{s})$;

$v_{c} \quad$ cutting velocity $(\mathrm{m} / \mathrm{s})$;

$w \quad$ kerf width $(\mathrm{m})$;

$Z \quad$ depth from nozzle exit $(\mathrm{mm})$;

$\rho \quad$ density of the gas $\left(\mathrm{kg} / \mathrm{m}^{3}\right)$;

$\rho_{m} \quad$ density of molten metal $\left(\mathrm{kg} / \mathrm{m}^{3}\right)$;

$\mu \quad$ gas $\left(\mathrm{O}_{2}\right)$ viscosity $\left(\sim 1.7 \times 10^{-5} \mathrm{~kg} / \mathrm{ms}\right)$;

$\mu_{m} \quad$ melt viscosity $\left(\sim 4 \times 10^{-3} \mathrm{~kg} / \mathrm{ms}\right)$;

$\tau \quad$ shear stress of the gas $\left(\mathrm{N} / \mathrm{m}^{2}\right)$;

$\Delta \quad$ melt film thickness (m).

\section{References}

Heidenreich B, Juptner W, Sepold G 1996 Fundamental investigation of the burn-out phenomenon of laser cut edges. Lasers Eng. 5: 1-10

Kaplan A F H 1996 An analytical model of metal cutting with a laser beam. J. Appl. Phys. 79: 21982207

Kumar K L 1988 Engineering fluid mechanics 4th edn (New Delhi: Eurasia)

Landau L D, Lifshitz E M 1987 Fluid mechanics 2nd rev. edn (Oxford: Butterworth-Heinemann)

Liepmann H W, Roshko A 1957 Elements of gas dynamics (New York: John Wiley \& Sons)

Shapiro H A 1953 The dynamics \& thermodynamics of compressible fluid flow (New York: John Wiley \& Sons)

Vicanek M, Simon G 1987 Momentum and heat transfer of an inert gas jet to the melt in laser cutting. J. Phys. D20: 1191-1196

Yilbas B S, Kar A 1998 Thermal and efficiency analysis of $\mathrm{CO}_{2}$ laser cutting process. Opt. Lasers Eng. 29: 17-32 